\title{
Metabolismo óseo, osteoporosis y enfermedad inflamatoria intestinal en adultos
}

\section{Bone Metabolism and Osteoporosis in Adults with Inflammatory Bowel Disease}

Duque $J J^{1}$, Moreno DC ${ }^{1}$, Arteaga $J M^{1,2}$, Franco $R^{1,2}$

${ }^{1}$ Unidad de Endocrinología, Universidad Nacional de Colombia, sede Bogotá

${ }^{2}$ Hospital Universitario Nacional

Fecha de recepción: 12/04/2019

Fecha de aceptación: 24/04/2019

\section{Resumen}

La osteoporosis secundaria es la disminución de la densidad mineral ósea y la alteración en la microarquitectura del tejido óseo, que conlleva un aumento en el riesgo de fracturas, y que es debida a factores diferentes a la edad y la postmenopausia.

Los pacientes con enfermedad inflamatoria intestinal tienen una compleja interacción de factores que propicia la aparición de osteoporosis. La identificación correcta de los mecanismos desencadenantes ha sido un reto, teniendo en cuenta la diversidad de medicamentos, condiciones y enfermedades que convergen en el paciente con una enfermedad inflamatoria intestinal. Entender los factores de riesgo y la importancia de mecanismos fisiopatológicos puede permitir un mejor enfoque preventivo y terapéutico.

Se presenta el caso de una mujer de 59 años con colitis ulcerativa desde los 18 años, manejada con múltiples cursos de esteroides, con signos clínicos y paraclínicos de malnutrición. La paciente asiste por dolor lumbar, lo que lleva al diagnóstico de fracturas vertebrales. Se describen múltiples mecanismos causales de osteoporosis en la paciente, incluida falla ovárica prematura, deficiencia de vitamina $\mathrm{D}$, desnutrición y uso de esteroides.

Esta revisión se centra en la fisiopatología de la osteoporosis secundaria a enfermedad inflamatoria intestinal y su abordaje diagnóstico y terapéutico en el contexto de un caso ilustrativo.

Palabras clave: osteoporosis, enfermedad inflamatoria intestinal (EII), enfermedad de Crohn, colitis ulcerativa, absorciometría de rayos X de energía dual (DXA).

\section{Abstract}

Secondary osteoporosis consists in the decreased bone mineral density and the alteration of the bone microarchitecture, due to factors other than age and post menopause. These changes lead to an increase in the risk of fragility and subsequent bone fractures. Patients with inflammatory bowel disease have a complex interaction of factors that favor the development of osteoporosis. The identification of the mechanisms triggering osteoporosis in these patients has been a challenge, considering the variety of drugs, conditions and illnesses that merge in the patient with an intestinal inflammatory disease. Understanding risk factors and the importance of physiopathological mechanisms can allow a better preventive and therapeutic approach.

The case of a woman of 59 Years with Ulcerative Colitis since age 18, is presented. The patient was managed with multiple steroid courses, with clinical and paraclinical signs of malnutrition. The patient presented with lumbar pain, which lead to the diagnosis of vertebral fractures. Multiple causal mechanisms of osteoporosis are described in the patient, including premature ovarian failure, vitamin D deficiency, malnutrition and steroid use.

This review focuses on the pathophysiology of osteoporosis secondary to inflammatory bowel disease and its diagnostic and therapeutic approach in the context of an illustrative case.

\section{Introducción}

La inflamación es un evento fisiopatológico con repercusiones en todos los órganos y sistemas. El impacto de las enfermedades inflamatorias sistémicas en el metabolismo óseo está ampliamente documentado. Las enfermedades inflamatorias intestinales (EII) corresponden a un espectro clínico caracterizado por inflamación crónica, malabsorción y malnutrición que afecta extensamente la salud de quienes lo padecen. Tanto los eventos que ocurren en esta enfermedad como su tratamiento (que con frecuencia requiere glucocorticoides) impacta de forma significativa la salud ósea ${ }^{(1)}$. 
El objetivo de este artículo es revisar el impacto, los mecanismos y posibles implicaciones terapéuticas de la osteoporosis en la EII, a través de un caso ilustrativo.

\section{Caso clínico}

Presentamos el caso de una mujer de 59 años con antecedentes de colitis ulcerativa diagnosticada desde los 18 años, manejada con mesalazina y ciclos intermitentes de glucocorticoides, insuficiencia ovárica prematura a los 34 años, sin terapia de reemplazo hormonal y tromboembolismo pulmonar diagnosticado hace seis meses en anticoagulación con heparina de bajo peso molecular, quien ingresa por cuadro clínico de dos meses de evolución de dolor lumbar intensidad 10/10, asociado con deposiciones diarreicas aproximadamente 20 al día.

A la revisión por sistemas, pérdida de altura de $4 \mathrm{~cm}$ a lo largo de la vida. Al examen físico de ingreso, bajo índice de masa corporal (IMC 17,3 Kg/m²), dolor a la palpación y percusión vertebral y paravertebral, sin cifoescoliosis evidente, sin otros hallazgos de importancia.

En los paraclínicos iniciales se evidencian fracturas vertebrales por insuficiencia en L2, L4 y L5, en radiografía simple de columna (figura 1), que se caracterizaron con resonancia magnética (figura 2). En el estudio de osteoporosis secundaria, se descarta hiperparatiroidismo, alteración del perfil tiroideo, hepático y renal. No se evidencia pico monoclonal en la electroforesis de proteínas. Se encuentra hipoalbuminemia y perfil fosfocálcico con déficit de vitamina D (tabla). Por lo que se considera osteoporosis secundaria a enfermedad inflamatoria intestinal. Se inicia manejo interdisciplinario con nutrición, terapia física, clínica del dolor y gastroenterología. Se inicia acompañamiento nutricional y rehabilitación física, suplencia de calcio y vitamina D y teriparatida, con mejoría de la sintomatología.

\section{Dimensión del problema}

Refiriéndose a EII usualmente hablamos del espectro comprendido entre enfermedad de Crohn y colitis ulcerativa. La incidencia global en países desarrollados oscila entre 7 y 20 casos por 100.000 habitantes por año, con una prevalencia entre 100 y 200 casos por 100.000 habitantes $^{(2)}$. Las últimas guías colombianas de la Asociación Colombiana de Gastroenterología, no presentan epidemiología local por la falta de datos que permitan calcular de forma confiable la epidemiología de $\mathrm{EII}^{(2)}$. De una forma más general, el Instituto de Métricas de Salud (IHME), que compila fuentes abiertas a nivel mundial para el cálculo de carga global de la enfermedad, estima una prevalencia general de EII de 27,78 casos por 100.000 habitantes, cifra algo menor que los 31,73 casos por 100.000 habitantes para la región de Latinoamérica y el Caribe, considerados por este mismo órgano ${ }^{(3)}$. De forma similar, en 2018 una revisión sistemática desarrollada en conjunto con el grupo Cochrane para Argentina evaluó la epidemiología de la EII en Latinoamérica y en ella se obtuvo un rango de incidencia
Figura 1. Fractura bicóncava de vértebra L2 con pérdida de aproximadamente $20 \%$ de la altura del cuerpo vertebral. Fractura por acuñamiento anterior de vértebras L3, L4 y L5 con pérdida de aproximadamente $10 \%, 30 \%$ y $25 \%$ de la altura de los cuerpos vertebrales, respectivamente. Cambios espondilóticos multinivel con formación de osteofitos marginales laterales en la columna lumbar. Cambios por artrosis facetaria y signos de osteocondritis multinivel más prominente en L2-L3. Anterolistesis de L5/S1 grado I por espondilólisis.

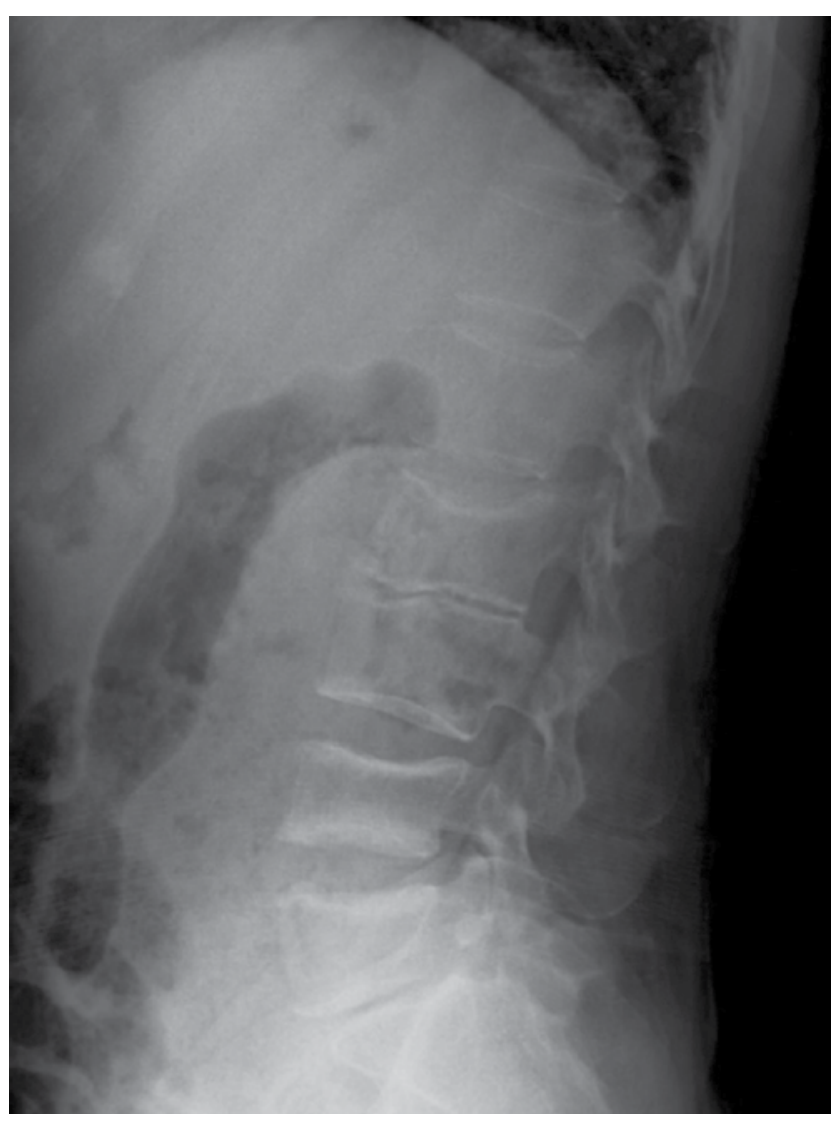

entre 0,74 y 6,75 por 100.000 personas año y una prevalencia que oscila entre 0,99 y $44 ., 3$ por 100.000 habitantes para colitis ulcerativa y entre 0,23 a 0,40 por 100.000 para enfermedad de Crohn, anotando una marcada variabilidad y pobre calidad de los datos encontrados ${ }^{(4)}$.

La osteoporosis es una complicación reconocida en la epidemiología de las EII. Aunque la evidencia al respecto es dispersa y en su mayoría basada en estudios transversales, estudios de comienzos de la década pasada sugieren una prevalencia de osteoporosis por DXA de $18 \%$ a $42 \%$, siendo aparentemente más común en la enfermedad de Crohn que en la colitis ulcerativa $^{(5,6)}$. En 2018, Szafors P. et al. realizaron una revisión sistemática con metanalisis para evaluar el riesgo de fracturas y baja densidad mineral ósea en pacientes con EII. En este estudio, los autores encontraron un aumento significativo del riesgo global de fracturas (RR 1,38 IC95\% 1,11-1,73) aunque la heterogeneidad de los estudios incluidos limita la 
Figura 2. Las secuencias T1 y T2 configuración bicóncava de los cuerpos vertebrales con pérdida de altura e hipointensidad de la médula ósea por fracturas antiguas de cuerpos vertebrales de L4 y L5. Osteocondrosis desde L2-L4 hasta L5-S1 con deshidratación de discos intervertebrales y pérdida de su altura. Fractura antigua de cuerpo vertebral T11 con depresión de platillo vertebral superior, discreto acuñamiento anterior y nódulo Schmorl. La secuencia STIR sugiere que las fracturas son antiguas, aunque hay discreta hiperintensidad en L4 que podría corresponder a edema óseo.

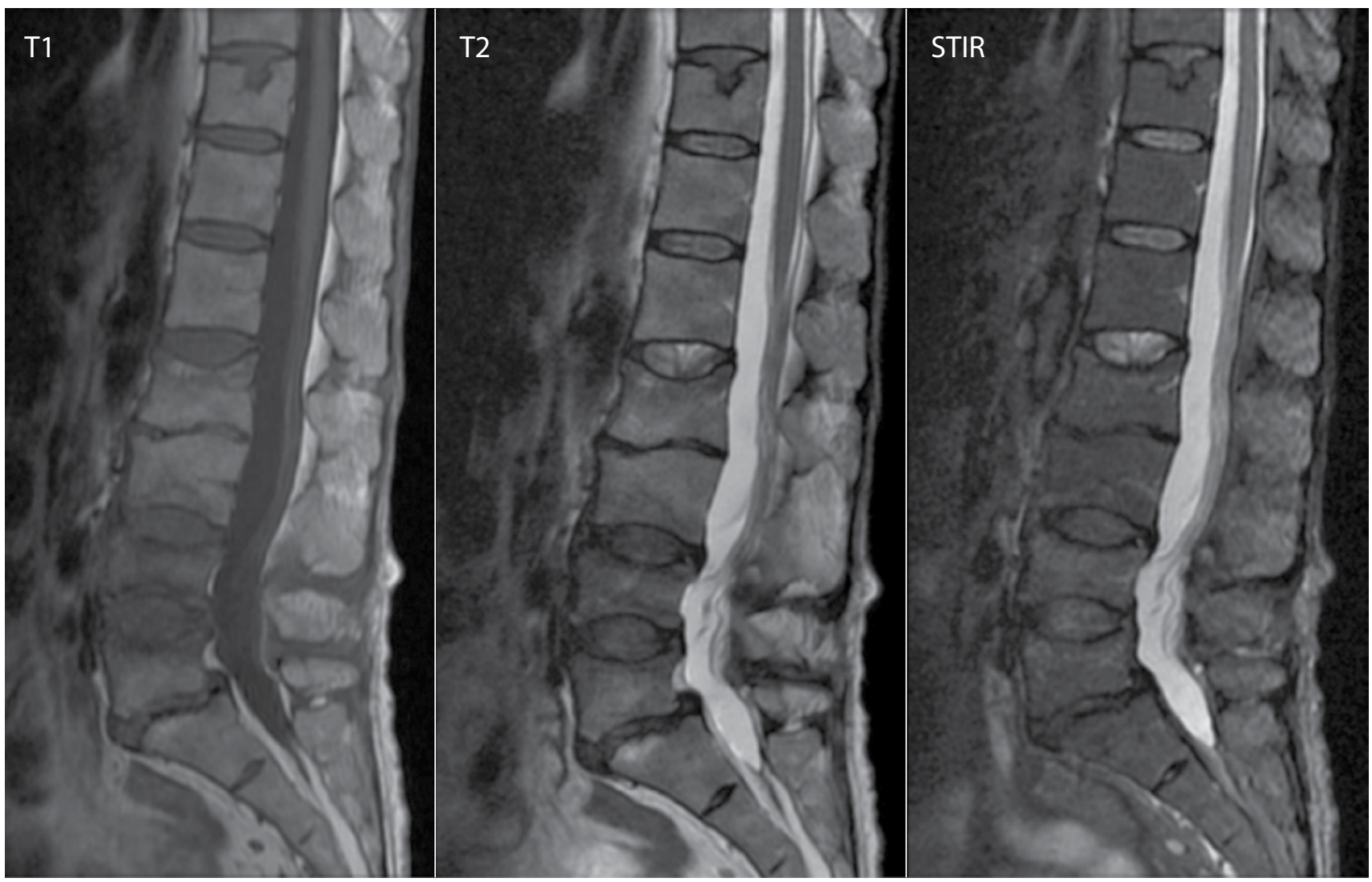

Tabla 1. Resultados de laboratorio

\section{Calcio colorimétrico corregido por albúmina $9,76 \mathrm{mg} / \mathrm{dl}$ Fósforo: $3,62 \mathrm{mg} / \mathrm{dl}, \mathrm{PTH}: 28,5 \mathrm{pg} / \mathrm{ml}$}

25 hidroxivitamina D: $9,27 \mathrm{ng} / \mathrm{ml}$

Proteínas totales en suero: $6,2 \mathrm{~g} / \mathrm{dl}$, albúmina: $2,8 \mathrm{~g} / \mathrm{dl}$

TSH: $0,757 \mu \mathrm{Ul} / \mathrm{ml}$

Bilirrubina total: $0,17 \mathrm{mg} / \mathrm{dl}, \mathrm{TGO}: 27,9 \mathrm{UI} / \mathrm{L}, \mathrm{TGP}: 35,1 \mathrm{UI} / \mathrm{L}$, GGT: 39 UI/L,

Fosfatasa alcalina: $115,0 \mathrm{UI} / \mathrm{L}$

PCR: $15,39 \mathrm{mg} / \mathrm{L}, \mathrm{VSG}: 78 \mathrm{~mm} / \mathrm{h}$

\section{Hemograma:}

Leucocitos: $4,08 \times 10^{3} / \mu \mathrm{L}$, neutrófilos: $2,43 \times 10^{3} / \mu \mathrm{L}$, linfocitos: $1,36 \times 10^{3} / \mu \mathrm{L}$,

Hematocrito: 37,7\%, Hemoglobina: 12,7g/dl, Plaquetas: 269 $\times 10^{3} / \mu \mathrm{L}$

\section{Electroforesis de proteínas sin pico monoclonal}

Creatinina: 0,64 mg/dl, BUN: 5,5 mg/dl interpretación de estos resultados. Respecto a la localización de las fracturas, el sitio más asociado a fracturas en pacientes con EII ha sido la columna lumbar, con un OR para fracturas vertebrales de 2,26 (IC95\% 1,04-4,90), mientras que el riesgo de fracturas extravertebrales no ha sido significativo en la mayoría de $\operatorname{series}^{(7)}$.

Pese a la falta de evidencia de riesgo de fracturas fuera de la columna, las medidas densitométricas son significativamente peores en EII, tanto en columna lumbar como en cuello femoral y fémur total, con una diferencia de medias de densidad mineral ósea de $-0,06 \mathrm{~g} / \mathrm{cm}^{2},-0,08 \mathrm{~g} / \mathrm{cm}^{2}$ y $0,06 \mathrm{~g} / \mathrm{cm}^{2}$ y un puntaje $\mathrm{Z}$ de $-0,52,-1,01$ y $-0,45$, respectivamente ${ }^{(7)}$. Si bien se desearía contar con cohortes prospectivas más recientes (especialmente en la era de los anti-TNF), se puede aceptar que el riesgo de osteoporosis en esta población está aumentado.

Entre los factores de riesgo reconocidos están: la carga inflamatoria, el uso de glucocorticoides, la deficiencia de vitamina D, el consumo de tabaco y la desnutrición ${ }^{(8)}$. Adicionalmente, algunos pacientes con enfermedad de Crohn pueden tener baja densidad mineral ósea desde el momento del diagnóstico, e incluso se ha reportado la osteoporosis como manifestación 
inicial de esta patología, lo que refuerza el rol de la inflamación como factor de riesgo esencial para su desarrollo ${ }^{(9)}$.

\section{Fisiopatología}

El tracto gastrointestinal es un sistema que, más allá de la absorción de nutrientes, funciona como una barrera inmune sólida que protege contra patógenos del ambiente y de la flora normal. De esta forma, cuando la homeostasis de la barrera inmune gastrointestinal se altera, pueden resultar patologías sistémicas diversas que van desde la sepsis hasta trastornos funcionales, inmunes o metabólicos. Las EII son la manifestación final de un proceso autoinmune sistémico, que se manifiesta como episodios recurrentes de inflamación intestinal con diferentes grados de severidad y compromiso preferente de algunas zonas del tracto gastrointestinal $^{(10)}$. En la enfermedad de Crohn, la inflamación afecta la totalidad de la pared intestinal y puede encontrarse en cualquier segmento del tubo digestivo. Por otro lado, la colitis ulcerativa afecta las capas más superficiales del colon ${ }^{(9,11)}$. La respuesta más característica de la enfermedad de Crohn consiste en la activación inflamatoria Th1, mientras en la colitis ulcerativa se reconoce un mayor rol de los linfocitos Th2. En ambas enfermedades, la activación de células Th17 reguladoras se reconoce como un componente indispensable para la perpetuación de la inflamación. De forma similar, la secreción de TNF- $\alpha$ es un factor común a ambas entidades, lo que ha sido corroborado con el éxito de terapias dirigidas a esta citoquina ${ }^{(10)}$.

Si bien, tanto la colitis ulcerativa como la enfermedad de Crohn se asocian a osteoporosis, la enfermedad de Crohn suele tener un componente inflamatorio sistémico mayor, que se asocia con peor arquitectura ósea ${ }^{(1,11)}$.

Estudios de arquitectura y geometría ósea han sido desarrollados con tomografía computarizada cuantitativa periférica (pQCT). En estos modelos, la enfermedad de Crohn ha mostrado disminución significativa de volumen de hueso trabecular y expansión de la superficie endocortical. De forma similar, la circunferencia periostial suele estar disminuida. Estos cambios sugieren tanto aumento de la resorción como disminución de la formación ósea ${ }^{(11)}$.

Los mecanismos propuestos sugieren que la inflamación crónica a nivel intestinal se asocia a producción de citoquinas proinflamatorias por parte de linfocitos T. Dentro de estas, el TNF- $\alpha$ es conocido por su capacidad de activar la vía del RANKRANK-ligando (RANKL), siendo el receptor RANK un miembro de la superfamilia de receptores de TNF. El resultado de esta interacción resulta en la diferenciación de los precursores de osteoclastos. Este aumento de la actividad del RANK resulta en una disminución relativa de osteoprotegerina (OPG), lo que resulta en un aumento de la resorción ${ }^{(12)}$. De forma paralela, el TNF- $\alpha$ se asocia a producción de esclerostina con disminución de la formación ósea, lo que inclina aún más la balanza hacia la resorción ${ }^{(1)}$.
El uso de corticoesteroides es uno de los factores que más impacto tiene en la osteoporosis de la EII. Se estima que aproximadamente el $50 \%$ de los pacientes diagnosticados con EII son expuestos a glucocorticoides sistémicos dentro de los primeros cinco años de su enfermedad, y al menos $20 \%$ han usado más de $3 \mathrm{~g}$ acumulados en un año. El mecanismo de la osteoporosis y el uso de glucocorticoides ocurre a varios niveles. Mientras las dosis acumuladas tienen un mayor impacto en el desarrollo de arquitectura defectuosa, las dosis altas tienen acción directa en la función del osteoblasto y osteocito, promoviendo el desarrollo de fracturas, aunque la masa ósea no esté disminuida ${ }^{(13)}$. Modelos murinos han demostrado que los glucocorticoides impiden la diferenciación de células madre mesenquimales hacia nuevos osteoblastos, y reducen el tiempo de vida de osteoblastos y osteocitos por inducción de apoptosis ${ }^{(8)}$. Los efectos en diferenciación parecen ser mediados por inhibición de la vía de Wnt, lo que se traduce en menor formación de osteoide, menor cantidad de osteoblastos y una prolongación de la fase de reversa del ciclo de remodelación ${ }^{(13)}$. Por otro lado, se ha propuesto que el uso crónico de corticoesteroides aumenta la expresión de RANKL y disminuye la expresión de osteoprotegerina en células estromales y osteoblásticas. Este desequilibrio promueve la diferenciación de células madre hematopoyéticas a osteoclastos y subsecuente resorción ósea, mientras se inhibe la síntesis de nueva matriz ósea. Eventualmente, predomina el efecto sobre la osteoblastogénesis, lo que hace que la disminución en la síntesis ósea sea más importante que el efecto en la resorción, lo que resulta en una osteoporosis de bajo recambio ${ }^{(8)}$. Es posible que el efecto de los glucocorticoides en las hormonas sexuales también juegue un papel importante, dado su efecto negativo en la producción de esteroides gonadales, con efectos similares como los que se producen en la menopausia donde hay osteoporosis por deficiencia de estrógenos ${ }^{(8)}$. Como efecto adicional, los glucocorticoides reducen la absorción intestinal de calcio y aumentan la excreción renal del mismo ${ }^{(14)}$.

También se ha propuesto la malabsorción intestinal como paso esencial en la fisiopatología de la osteoporosis de la EII. Los argumentos incluyen la reducida absorción de vitamina D y calcio, por inflamación en intestino delgado, y disminución de reabsorción de ácidos biliares (y en consecuencia de vitamina D) por inflamación en el íleon terminal. La deficiencia de vitamina $\mathrm{D}$ es un factor predisponente de osteoporosis por su efecto en la absorción de calcio, y acción directa sobre osteoblastos. Cuando la vitamina D está baja, se estimulan mecanismos compensatorios para mantener la calcemia. Uno de estos mecanismos es el aumento de hormona paratiroidea, que tiene acción directa en su receptor en el osteoblasto y resulta en una expresión aumentada de RANKL, lo que a su vez estimula la diferenciación de osteoclastos y promueve resorción ósea por los mecanismos ya descritos ${ }^{(8)}$.

Un factor de interés para la osteoporosis en la EII es la sarcopenia. Recientemente se ha descrito cómo la baja masa muscular es una condición prevalente en los pacientes con EII 
y esta suele asociarse a mayor severidad de terapia de rescate para EII ${ }^{(15)}$. Como es de esperarse, la sarcopenia parece ser un factor predictivo para baja masa ósea ${ }^{(16,17)}$.

Se ha demostrado que la disminución en la masa y fuerza muscular secundaria a factores nutricionales, sedentarismo y uso crónico de corticoides puede conllevar una disminución en la masa ósea por una reducción en las cargas mecánicas sobre el esqueleto ${ }^{(8)}$. Por lo tanto, la composición ósea parece ser más relevante que el índice de masa corporal en el metabolismo óseo de los pacientes con EII.

Si bien la desnutrición y el bajo índice de masa corporal actúan en detrimento de la salud ósea, la obesidad en EII puede ser igualmente problemática. La ganancia de peso hacia el sobrepeso y obesidad en pacientes con EII se asocia con ganancia de índices de grasa visceral y pérdida de masa muscular, con mayores tasas de sarcopenia. Esta miopenia puede perpetuar el ciclo antes descrito de sarcopenia/osteopenia en el paciente con $\mathrm{EII}^{(18)}$.

Si bien el tabaquismo no es un factor intrínseco de las EII, es importante mencionarlo, puesto que algunos estudios sugieren una mayor prevalencia, particularmente en pacientes con colitis ulcerativa. En esta patología, el consumo de cigarrillo se ha asociado consistentemente a reducción de frecuencia y severidad de las crisis, así como un menor uso de esteroides; mientras que el cese de tabaco puede empeorar el curso clínico $^{(19,20)}$. En enfermedad de Crohn el tabaquismo tiene el efecto contrario y no parece haber más riesgo de fumar que con la población general ${ }^{(19)}$. Si bien el cigarrillo reduce el uso de esteroides en colitis ulcerativa su efecto neto promueve la pérdida ósea.

\section{Enfoque preventivo}

En enfermedad inflamatoria intestinal se deben tamizar los pacientes con alto riesgo de osteoporosis. Algunas sociedades científicas han publicado recomendaciones acerca de la tamización y tratamiento de osteoporosis en enfermedad inflamatoria intestinal. Los últimos consensos de la Asociación Americana de Gastroenterología (AGA) y Sociedad Británica de Gastroenterología se publicaron en 2003 y 2007, respectivamente. Ambas guías concuerdan en tamizar con DXA a los pacientes de alto riesgo, pero no de rutina en todos los pacientes con EII. Entre los factores de alto riesgo en que justificarían el tamizaje con DXA se aceptan: fractura por fragilidad previa independiente de la edad, postmenopausia, hombres mayores de 50 años, uso de corticoides durante más de tres meses, hipogonadismo.

Las guías clínicas de la ACG del 2017 recomiendan con un bajo nivel de evidencia que los pacientes con factores de riesgo convencionales para alteración en la densidad mineral ósea con EII deben ser tamizados en el momento del diagnóstico y periódicamente. Si la DXA inicial es normal, la AGA recomienda repetirla cada 2 a 3 años ${ }^{(21)}$.

Por otro lado, otros factores como consumo de alcohol, tabaquismo, bajo peso, sedentarismo también deben ser consi- derados ${ }^{(6,22)}$. Finalmente, como se dijo antes, la enfermedad de Crohn tiene una carga inflamatoria más alta y puede presentar osteoporosis desde su diagnóstico; por tanto, debe haber un umbral más bajo para ordenar densitometría.

\section{Implicaciones terapéuticas}

El tratamiento de la EII ha cambiado dramáticamente en la era de los medicamentos anti TNF. Desde el punto de vista teórico se presume que la inhibición de TNF- $\alpha$ tendría un efecto benéfico en el metabolismo óseo, sin embargo, los resultados que respaldan esta idea son variados. Algunos estudios han mostrado que el tratamiento con infliximab se asocia con efectos benéficos en el metabolismo óseo, con mejoría de la viabilidad de osteoblastos, con mejoría de niveles de osteoformación (como osteocalcina, fosfatasa alcalina) y disminución de niveles de resorción (como $\beta$-Cross Laps) ${ }^{(8,23)}$. Estudios retrospectivos han mostrado mejoría de densidad y contenido mineral óseo. Sin embargo, no hay estudios prospectivos evaluando desenlaces clínicos de la terapia biológica en la osteoporosis del paciente con EII.

Entre las medidas no farmacológicas, las recomendaciones son las mismas que para las mujeres con osteoporosis postmenopáusica. En general, se recomienda una evaluación y asesoría sobre el riesgo de caídas, ejercicio de fortalecimiento y mejoramiento de balance, abandonar el hábito tabáquico y reducir el consumo de alcohol. Dentro del manejo farmacológico se recomienda la evaluación de niveles de vitamina $\mathrm{D}$ y suplementación en caso de ser necesario ${ }^{(24)}$.

Tanto la AGA como la sociedad británica de gastroenterología recomiendan que los pacientes con riesgo de osteoporosis deben ser suplementados con calcio y vitamina D, para hombres jóvenes y mujeres premenopáusicas para alcanzar un consumo diario de calcio elemental de $1000 \mathrm{mg}$ y hombres y mujeres mayores de 50 años de $1500 \mathrm{mg}$ en la dieta o suplementos y consumo mucho mayor de vitamina D a lo recomendado para individuos sanos ${ }^{(14)}$.

Existen múltiples medicamentos antirresortivos y anabólicos que han demostrado ser efectivos y seguros para el tratamiento de la osteoporosis y la prevención de fracturas; sin embargo, no hay suficiente literatura para determinar un tratamiento farmacológico específico para el manejo de pacientes con EII diferente al establecido para pacientes con osteoporosis por cualquier otra causa ${ }^{(25)}$.

Los bifosfonatos son tal vez los medicamentos más ampliamente estudiados para el tratamiento de osteoporosis. Por lo que se ha demostrado que el ácido zoledrónico es la mejor estrategia terapéutica para la mejoría de la densidad mineral ósea, mientras que el risedronato tuvo la menor cantidad de efectos adversos en un metanalisis realizado con pacientes con enfermedad de Crohn ${ }^{(26)}$. Sin embargo, hacen falta más estudios con los diferentes grupos de medicamentos en esta población específica. En su consenso más reciente, la Asociación Colombiana de Gastroenterología, reconoce la importancia de 
la osteopenia y osteoporosis como condiciones comórbidas de la EII, y sugiere un manejo conjunto con endocrinología. Por esto, esta guía recomienda considerar la suplementación de calcio y vitamina D, con la opción de uso de bifosfonatos en caso de diagnóstico de enfermedad metabólica ósea ${ }^{(2)}$.

Si bien los bifosfonatos han sido los medicamentos más estudiados en el manejo de la osteoporosis, el uso de otros grupos de medicamentos, como los homólogos de hormona paratiroidea, o los anticuerpos monoclonales contra el ligando de RANK o contra resistina como el romozosumab, no se descartan en pacientes con EII y alteración ósea ${ }^{(22)}$.

\section{Conclusiones}

La osteoporosis es una enfermedad prevalente que generalmente tiene causas identificables. Una etiología importante pero no siempre investigada es la enfermedad inflamatoria intestinal. Un espectro de enfermedades que no solo implica una condición inflamatoria crónica, sino también un síndrome malabsortivo con desnutrición secundaria y el uso repetido de corticoides para su control. El adecuado conocimiento de su fisiopatología nos permite un acertado abordaje diagnóstico y terapéutico.

En este caso descrito podemos ver los múltiples factores que interactúan en la EII para el desarrollo de osteoporosis y la complejidad de su fisiopatología.
Se hace indispensable un cribado adecuado y sistemático para osteoporosis en los pacientes con enfermedad inflamatoria intestinal, así como un manejo oportuno de los factores modificables que pueden estar asociados con la disminución de la masa ósea en estos pacientes, como lo son un bajo índice de masa corporal, aporte inadecuado de calcio y vitamina D, la disminución en la actividad física, el hipogonadismo que puede estar asociado y el uso crónico de glucocorticoides sistémicos, para evitar un aumento en la morbimortalidad propio de las fracturas por fragilidad.

En el caso descrito se requirió un manejo multidisciplinario por el servicio de gastroenterología para control de su enfermedad de base, nutrición y rehabilitación física para intervención de desnutrición, bajo índice de masa corporal y muscular, clínica del dolor y endocrinología, para suplencia de calcio y vitamina D, así como uso de teriparatida, teniendo en cuenta la presencia de fracturas vertebrales, con disminución notable del dolor óseo.

\section{Declaración de fuentes de financiación y posibles conflic- tos de interés}

Los autores no recibieron fondos para la realización del presente trabajo.

\section{Referencias}

1. Briot K, Geusens P, Em Bultink I, Lems WF, Roux C. Inflammatory diseases and bone fragility. Osteoporos Int. 2017 Dec 15;28(12):3301-14.

2. Aguirre D, Archila PE, Carrera J, Castaño R, Escobar CM, Garcia, et al. Consenso colombiano de enfermedad inflamatoria intestinal. Rev Colomb Gastroenterol. Asociación Colombiana de Gastroenterolog $\backslash \backslash \backslash \mathrm{i}\} \mathrm{a}$; 2012;27:s1-s44.

3. IHME. GBD Compare Data Visualization [Internet]. Seattle, WA: Institute for Health Metrics and Evaluation, University of Washington. 2016 [cited 2019 Mar 25]. Available from: http://vizhub.healthdata.org/gbd-compare

4. Calderón M, Minckas N, Nuñez S, Ciapponi A. Inflammatory Bowel Disease in Latin America: A Systematic Review. Value Heal Reg Issues. 2018 Dec;17:126-34.

5. van Hogezand RA, Hamdy NAT. Skeletal morbidity in inflammatory bowel disease. Scand J Gastroenterol Suppl. 2006 Jan 8;41(243):59-64.

6. Bernstein CN, Leslie WD, Leboff MS. AGA technical review on osteoporosis in gastrointestinal diseases. Gastroenterology. 2003 Mar;124(3):795-841.

7. Szafors P, Che H, Barnetche T, Morel J, Gaujoux-Viala C, Combe B, et al. Risk of fracture and low bone mineral density in adults with inflammatory bowel diseases. A systematic literature review with meta-analysis. Osteoporos Int. 2018 Nov 16;29(11):2389-97.

8. Lima CA, Lyra AC, Rocha R, Santana GO. Risk factors for osteoporosis in inflammatory bowel disease patients. World J Gastrointest Pathophysiol. Baishideng Publishing Group Inc; 2015 Nov 15;6(4):210-8.

9. Wei H, Ouyang C, Peng D, Lu F, Zhang J. Osteoporosis as an initial manifestation in a patient with Crohn's disease: A case report. Exp Ther Med. Spandidos Publications; 2018 Apr;15(4):3997-4000.

10. Kim DH, Cheon JH. Pathogenesis of Inflammatory Bowel Disease and Recent Advances in Biologic Therapies. Immune Netw. The Korean Association of Immunologists; 2017 Feb;17(1):25-40.

11. Sylvester FA. Inflammatory bowel disease: Effects on bone and mechanisms. In: McCabe L, Parameswaran N, editors. Advances in Experimental Medicine and Biology. Springer; 2017. p. 133-50.

12. Lima CA. Risk factors for osteoporosis in inflammatory bowel disease patients. World J Gastrointest Pathophysiol. 2015;6(4):210.

13. Saag K, Adler RA. Glucocorticoid-Induced Osteoporosis. In: Primer on the Metabolic Bone Diseases and Disorders of Mineral Metabolism. Wiley; 2018. p. 467-73.
14. Ali T, Lam D, Bronze MS, Humphrey MB. Osteoporosis in Inflammatory Bowel Disease. Am J Med. 2009 Jul;122(7):599-604.

15. Cushing KC, Kordbacheh H, Gee MS, Kambadakone A, Ananthakrishnan AN. Sarcopenia is a Novel Predictor of the Need for Rescue Therapy in Hospitalized Ulcerative Colitis Patients. J Crohn's Colitis. 2018 May 12;

16. Bryant R V., Trott MJ, Bartholomeusz FD, Andrews JM. Systematic review: body composition in adults with inflammatory bowel disease. Aliment Pharmacol Ther. 2013 Aug;38(3):213-25.

17. Bryant R V., Ooi S, Schultz CG, Goess C, Grafton R, Hughes J, et al. Low muscle mass and sarcopenia: common and predictive of osteopenia in inflammatory bowel disease. Aliment Pharmacol Ther. 2015 May;41(9):895-906.

18. Bryant R, Schultz C, Ooi S, Goess C, Costello S, Vincent A, et al. Obesity in Inflammatory Bowel Disease: Gains in Adiposity despite High Prevalence of Myopenia and Osteopenia. Nutrients. 2018 Sep 1;10(9):1192.

19. Wang P, Hu J, Ghadermarzi S, Raza A, O'Connell D, Xiao A, et al. Smoking and Inflammatory Bowel Disease: A Comparison of China, India, and the USA. Dig Dis Sci. 2018 Oct 4;63(10):2703-13.

20. Parkes GC, Whelan K, Lindsay JO. Smoking in inflammatory bowel disease: Impact on disease course and insights into the aetiology of its effect. J Crohn's Colitis. Narnia; 2014 Aug 1;8(8):717-25.

21. Farraye FA, Melmed GY, Lichtenstein GR, Kane S V. ACG Clinical Guideline: Preventive Care in Inflammatory Bowel Disease. Am J Gastroenterol. 2017 Feb;112(2):241-58.

22. of Gastroenterology BS, Lewis NR, Scott BB. Guidelines for osteoporosis in inflammatory bowel disease and coeliac disease. British Society of Gastroenterology; 2008.

23. Franchimont N, Putzeys V, Collette J, Vermeire S, Rutgeerts P, De Vos M, et al. Rapid improvement of bone metabolism after infliximab treatment in Crohn's disease. Aliment Pharmacol Ther. 2004 Sep 15;20(6):607-14.

24. Tindell A, Johnsson H, McInnes IB. Arthritis, Arthropathy, and Osteoporosis in Inflammatory Bowel Disease. In: Crohn's Disease and Ulcerative Colitis. Cham: Springer International Publishing; 2017. p. 571-83.

25. Piodi LP. Managing osteoporosis in ulcerative colitis: Something new? World J Gastroenterol. 2014;20(39):14087.

26. Zhao X, Zhou C, Chen H, Ma J, Zhu Y, Wang P, et al. Efficacy and safety of medical therapy for low bone mineral density in patients with Crohn disease. Medicine (Baltimore). 2017 Mar;96(11):e6378. 